\title{
Fontes de radiação não ionizante e casos de leucemia infantil: uma revisão integrativa
}

\author{
Sources of non-ionizing radiation and cases of childhood leukemia: an integrative review \\ Fuentes de radiación no ionizante y casos de leucemia infantil: una revisión integradora
}

Recebido: 17/09/2021 | Revisado: 21/09/2021 | Aceito: 01/10/2021 | Publicado: 03/10/2021

\author{
Cyntia Maria Moreira Herkert \\ ORCID: https://orcid.org/0000-0002-8865-8530 \\ Faculdade Estácio Campo Grande, Brasil \\ E-mail: cyntia.herkert@estacio.br \\ Andréia Insabralde de Queiroz Cardoso \\ ORCID: https://orcid.org/0000-0002-9431-7484 \\ Universidade Federal de Mato Grosso do Sul, Brasil \\ E-mail: andreia.cardoso@ufms.br \\ Alexandra Maria Almeida Carvalho Pinto \\ ORCID: https://orcid.org/0000-0002-7868-3214 \\ Universidade Federal de Mato Grosso do Sul, Brasil \\ E-mail: profalexandraufms@gmail.com
}

\begin{abstract}
Resumo
Introdução: A leucemia infantil é considerada o câncer mais comum em crianças de 0 a 14 anos, e está relacionada a fatores genéticos e mais fortemente aos fatores ambientais, dentre os quais a radiação não ionizante de baixa frequência vem sendo estudada como fator de risco. Objetivo: Analisar evidências científicas sobre a associação entre fontes de radiação não ionizante de baixa frequência e a ocorrência de leucemia infantil. Metodologia: Trata-se de uma revisão integrativa onde os descritores utilizados foram Electromagnetic Fields and Leukemia, indexados no Medical Subject Headings, para pesquisa nas bases de dados PubMed, Elsevier's Scopus and Web of Science, e Magnetic Fields and Leukemia, indexados nos descritores em Ciências da Saúde, para pesquisa na Biblioteca Virtual da Saúde. Dois pesquisadores selecionaram artigos completos de estudos de caso-controle publicados de 2010 a 2020. Dois pesquisadores selecionaram artigos completos sobre estudos de caso-controle publicados de 2010 a 2020 . O software Rayyan QCRI foi utilizado para análise dos artigos. Resultados: Foram analisados cinco artigos que atenderam ao delineamento metodológico proposto. Os artigos foram publicados em inglês, de 2012 a 2020, e os participantes dos estudos tinham idade inferior a 16 anos. Os desenhos dos métodos para avaliação da exposição foram heterogêneos, assim como o ambiente analisado. As limitações dos estudos foram decorrentes da ausência de avaliação de outras fontes externas potenciais ao desenvolvimento da leucemia infantil. Conclusão: Vale ressaltar que a exposição aos campos eletromagnéticos ocorre por diferentes fontes e os efeitos fisiológicos ainda precisam ser melhor explorados. Estudos robustos são necessários para analisar campos eletromagnéticos de baixa frequência como possível carcinogênico aos seres humanos. Em decorrência da heterogeneidade metodológica e de variáveis de confundimento existentes nos artigos analisados, os autores concluíram que não foi possível evidenciar a relação entre as fontes de radiação não ionizante de baixa frequência e o desenvolvimento da leucemia infantil.
\end{abstract}

Palavras-chave: Campo eletromagnético; Leucemia; Radiação não ionizante.

\begin{abstract}
Introduction: Childhood leukemia is considered the most common cancer in children from 0 to 14 years old and it is related to genetics factors and, more strongly, to environmental factors, among which low-frequency non-ionizing radiation has been studied as a risk factor. Objective: To analyze scientific evidence on the association between lowfrequency non-ionizing radiation sources and the occurrence of childhood leukemia. Methodology: Integrative review, whose terms were the descriptors Electromagnetic Fields and Leukemia, from Medical Subject Headings, for search in PubMed, Elsevier's Scopus and Web of Science, and Magnetic Fields and Leukemia, from Descriptors in Health Sciences, for search in the Virtual Health Library. Two researchers selected full articles from case-control studies published from 2010 to 2020. The Rayyan QCRI software was used to analyze the articles. Results: Five articles that met the proposed methodological design were analyzed. The articles were published in English, from 2012 to 2020, and study participants were under 16 years of age. The designs of the methods for assessing exposure were heterogeneous, as was the environment analyzed. The limitations of the studies were due to the lack of evaluation of other potential external sources for the development of childhood leukemia. Conclusion: It is noteworthy that exposure to electromagnetic fields occurs from different sources and the physiological effects still need to be better explored. Robust studies are needed to analyze low frequency electromagnetic fields as a possible carcinogen to
\end{abstract}


humans. Due to methodological heterogeneity and confounding variables in the analyzed articles, the authors concluded that it was not possible to demonstrate the relationship between low-frequency non-ionizing radiation sources and the development of childhood leukemia.

Keywords: Electromagnetic fields; Leukemia; Radiation, nonionizing.

\section{Resumen}

Introducción: La leucemia infantil es considerada el cáncer más común en niños de 0 a 14 años, y está relacionada con factores genéticos y más fuertemente con factores ambientales, entre los que se ha estudiado la radiación no ionizante de baja frecuencia como factor de riesgo. Objetivo: Analizar la evidencia científica sobre la asociación entre fuentes de radiación no ionizante de baja frecuencia y la ocurrencia de leucemia infantil. Metodología: Revisión integrativa, cuyos términos fueron los descriptores Campos electromagnéticos y leucemia, de Medical Subject Headings, para búsqueda en PubMed, Elsevier's Scopus y Web of Science, y Magnetic Fields and Leukemia, de Descriptors in Health Sciences, para búsqueda en Virtual Health. Biblioteca. Dos investigadores seleccionaron artículos completos de estudios de casos y controles publicados entre 2010 y 2020. Dos investigadores seleccionaron artículos completos de estudios de casos y controles publicados entre 2010 y 2020. Se utilizó el software Rayyan QCRI para analizar los artículos. Resultados: Se analizaron cinco artículos que cumplieron con el diseño metodológico propuesto. Los artículos se publicaron en inglés, de 2012 a 2020, y los participantes del estudio eran menores de 16 años. Los diseños de los métodos para evaluar la exposición fueron heterogéneos, al igual que el entorno analizado. Las limitaciones de los estudios se debieron a la falta de evaluación de otras posibles fuentes externas para el desarrollo de la leucemia infantil. Conclusión: Cabe señalar que la exposición a campos electromagnéticos se produce a partir de diferentes fuentes y los efectos fisiológicos aún deben explorarse mejor. Se necesitan estudios sólidos para analizar los campos electromagnéticos de baja frecuencia como un posible carcinógeno para los humanos. Debido a la heterogeneidad metodológica y las variables de confusión en los artículos analizados, los autores concluyeron que no era posible demostrar la relación entre las fuentes de radiación no ionizante de baja frecuencia y el desarrollo de leucemia infantil.

Palabras clave: Campo electromagnético; Leucemia; Radiación no ionizante.

\section{Introdução}

A leucemia é doença que acomete as células do sangue, possuí como principal característica o acúmulo de células doentes localizadas na medula óssea em substituição as células sanguíneas normais. Estas células doentes possuem funcionamento inadequado, multiplicam-se rapidamente e morrem em menor quantidade do que a célula normal. Para identificar qual o tipo de leucemia, será necessário identificar a célula sanguínea que se tornará cancerosa, o modo de crescimento, para a caracterização da doença em aguda ou crônica (INCA, 2019).

A estimativa de casos novos de leucemia calculados para o Brasil, para cada ano do triênio 2020-2022, equivale a 5.920 casos em homens e de 4.890 em mulheres. Os valores apresentados configuram um risco esperado de 5,67 casos novos a cada 100 mil homens e 4,56 para cada 100 mil mulheres. Possui maior prevalência nos adultos com idade superior a 55 anos, porém também encontrada em crianças menores de 15 anos (INCA, 2019).

Esta patologia é considerada o câncer mais comum em crianças de 0 a 14 anos, e representa cerca de um terço dos cânceres pediátricos (Whitehead et al., 2016). A classificação da leucemia infantil baseia-se no tipo celular afetado com predominância e nível de diferenciação celular. As terminologias mielóide e linfóide identificam a célula envolvida que podem se proliferar em formas agudas e crônicas. As principais são a leucemia linfocítica em $80 \%$ dos casos, a leucemia mieloide aguda em 25\% dos casos e a leucemia mieloide crônica em 5\% dos casos (Maia \& Filho, 2013; Coebergh et al., 2006).

Os casos de leucemia podem surgir em decorrência dos fatores genéticos como as fusões gênicas e translocações (Jin et al., 2016), os quais englobam uma pequena fração de menos de 10\% dos casos de leucemias infantis (Curtin et al., 2014). Ou podem ocorrer, também devido aos fatores ambientais que incluem radiação ionizante, hidrocarbonetos aromáticos policíclicos, bifenilos policlorados, éteres difenílicos polibromados, pesticidas, uso de álcool, tabagismo e uso de drogas ilícitas pelos familiares (Whitehead et al., 2016). 
Vale ressaltar que os fatores ambientais possuem significativo papel etiológico nas leucemias infantis, e eles possuem enorme importância no conhecimento acerca dos fatores de risco e a adoção de medidas preventivas que possam reduzir potencialmente as exposições prejudiciais e risco de desenvolvimento da doença (Belson et al., 2007).

Com base em estudos epidemiológicos sobre associação entre leucemia infantil e a exposição a frequência extremamente baixa de campos magnéticos (ELF-ML), houve a classificação deste como possível carcinogênico ambiental aos seres humanos - Grupo 2B (International Agency for Research on Cancer [IARC], 2002; Greenland et al., 2000). Fato este confirmado no ano de 2015 pelo Comité Científico da Comissão Europeia para os Riscos para a Saúde Emergentes e Recentemente Identificados (Scientific Committeeon Emerging Newly Identified Health Risks [CRSERI], 2015).

Nos últimos tempos, ocorreu aumento significativo na utilização de aplicativos e dispositivos que irradiam radiações não ionizantes, que podem ser nomeadas como campos eletromagnéticos, que podem ser exemplificados como distribuição e uso da eletricidade, bem como as tecnologias de informação e comunicação (Scarfi et al., 2019).

Os campos magnéticos de frequência baixa possuem longos comprimentos de ondas e envolvem faixa de $3 \mathrm{a} 3000 \mathrm{~Hz}$ e os campos de frequência extremamente baixa, ocupam as faixas de $50 \mathrm{a} 60 \mathrm{~Hz}$, gerados por fontes naturais e produzidas pelo homem (Marcilio et al., 2009). Evidências epidemiológicas mencionam uma correlação entre a exposição a estes campos eletromagnéticos e a incidência do câncer infantil, o que tem emergido potenciais preocupações na saúde pública (Karimi et al., 2020).

Os estudos epidemiológicos focam nos possíveis efeitos na saúde humana, com relação à exposição dos campos magnéticos de frequência extremamente baixa, em especial no desenvolvimento do câncer infantil (Pedersen et al., 2014). Wertheimer e Leeper (1979), realizaram um estudo pioneiro e demonstraram a relação entre populações que residiam próximas a linhas de energia (telefonia celular) e a incidência de leucemia infantil. Nas últimas décadas cresceu o interesse científico em estudar a associação entre campos eletromagnéticos de baixa frequência e risco de desenvolvimento do câncer humano, pois a intensidade de radiação eletromagnética no ambiente humano tem aumentado (Kaszuba-Zwoinsk et al., 2015).

Os seres humanos são expostos diariamente a campos eletromagnéticos, seja ao ar livre, no ambiente domiciliar e de trabalho. Esta exposição a longo prazo possui efeitos acumulativos que ocasionam danos no genoma humano, e podem levar ao desenvolvimento de doenças, como o câncer. Embora existam deficiências e contradições diante deste tema, não se pode ignorar os estudos. Se faz necessário futuras investigações precisas, com número expressivo de indivíduos e adequada análise estatística dos dados (Diab, 2020).

Esta revisão integrativa faz parte de uma pesquisa prospectiva que visa avaliar a relação entre a presença de fontes de radiação não ionizante, decorrentes da telefonia celular e a incidência de leucemia infantil. Assim, esta revisão objetiva analisar as evidências científicas referentes a associação entre a presença de fontes ELF-ML e a ocorrência de leucemia infantil.

\section{Metodologia}

Este estudo é uma revisão integrativa de literatura, onde foram seguidos os passos de identificação do problema, elaboração da questão norteadora, busca na literatura, aplicação de teste de relevância para seleção, coleta de dados por instrumento estruturado, análise e apresentação da revisão (Stillwell et al., 2010; Whittemore \& Knafl, 2005).

Foi adotado o acrômio PICOS (Donato \& Donato, 2019) para elaboração da questão norteadora, considerando P (população/contexto): População pediátrica; I (Intervenção): Exposição a fontes de radiação não ionizante; C (comparador): Ausência de exposição a fonte de radiação não ionizante; O (outcome): desenvolvimento de leucemia infantil; S (estudos): Observacionais de Caso Controle. Mediante a estratégia construída, a questão norteadora foi definida como: "Qual a 
associação existente entre a presença de fontes de radiação não ionizante de frequência extremamente baixa e a ocorrência de leucemia infantil?".

A coleta de dados ocorreu em abril de 2021, com protocolo estruturado especificamente para esta pesquisa. A estratégia de busca ocorreu com a utilização dos descritores controlados "Electromagnetic Fields" e "Leukemia" indexados no Medical Subject Headings (MeSH), para busca nas bases de dados eletrônicas PubMed, Elservier's Scopus (SCOPUS) e Web of Science. Similarmente em português, com os termos "Campos Magnéticos" e "Leucemia", Descritores em Ciências da Saúde (DECS), para busca na Biblioteca Virtual de Saúde (BVS). Foram incluídos artigos completos sobre estudos observacionais de caso controle, publicados de 2010 a 2020, em qualquer idioma. A estratégia de busca para cada base de dados está descrita no Quadro 1.

Quadro 1. Bases de dados e estratégias de busca para a revisão integrativa.

\begin{tabular}{|l|l|}
\hline \multicolumn{1}{|c|}{ Base de dados } & \multicolumn{1}{c|}{ Estratégia de Busca } \\
\hline Pubmed & 1\# (("Electromagnetic Fields"[Mesh]) AND "Leukemia"[Mesh]) \\
\hline Scopus & 1\# (("Electromagnetic Fields"[Mesh] ) AND "Leukemia"[Mesh] ) \\
\hline Web of Science & 1\# TS=(Electromagnetic Fields AND Leukemia) \\
\hline BVS & 1\# (Campos Magnéticos) AND (Leucemia) \\
\hline
\end{tabular}

Fonte: Autores.

Dois pesquisadores fizeram a seleção dos artigos de forma independente. O Software Rayyan QCRI (Ouzzani et al., 2016) foi utilizado no processo de análise, com a aplicação do teste de relevância, composto pelos critérios de inclusão e exclusão, leitura de título e resumos.

Inicialmente os artigos são analisados para exclusão de artigos duplicados e de publicações dos seguintes tipos: revisões, cartas ao editor, resumos e opiniões de especialistas. Na sequência, a amostra resultante é analisada pela leitura do título e resumo, para verificar pertinência ao tema da revisão, neste trabalho, estudos sobre análise da exposição a campos magnéticos de frequência extremamente baixa e risco de leucemia infantil. Foram excluídos todos os artigos referentes a linhas de alta tensão e outras categorias de radiação não a ionizante. Após esta etapa, os artigos selecionados foram lidos na íntegra para extração das variáveis de interesse. Os dados foram alocados em planilhas estruturadas no Microsoft Excel. Os resultados foram sintetizados e agrupados para a avaliação e interpretação dos achados por análise descritiva resultantes dos estudos em decorrência da heterogeneidade dos dados.

\section{Resultados e Discussão}

As buscas nas bases de dados resultaram em 1394 artigos científicos. A maior quantidade de artigos fora recuperada na Web of Science e Pubmed, com 867 e 447 artigos respectivamente; a menor quantidade na Scopus com 44 e BVS com 36 artigos.

Os 1394 artigos foram verificados para busca de repetição, sendo retiradas 199 duplicatas. Pela leitura de título e resumo, a revisão por pares descartou 1195 artigos por não atenderem aos critérios de inclusão do estudo (completo e período da pesquisa), ou não eram sobre o tema investigado neste trabalho. Após esta etapa, nove artigos foram lidos na íntegra, e dentre estes quatro foram excluídos em decorrência da exposição ser relativa a campos eletromagnéticos de alta frequência. Assim, a análise desta revisão integrativa foi realizada com cinco artigos, todos em inglês. A Figura 1 apresenta o fluxograma (Moher et al., 2009) com a descrição das etapas da revisão e o quantitativo de artigos em cada uma. 
Figura 1. Fluxograma de seleção dos estudos.

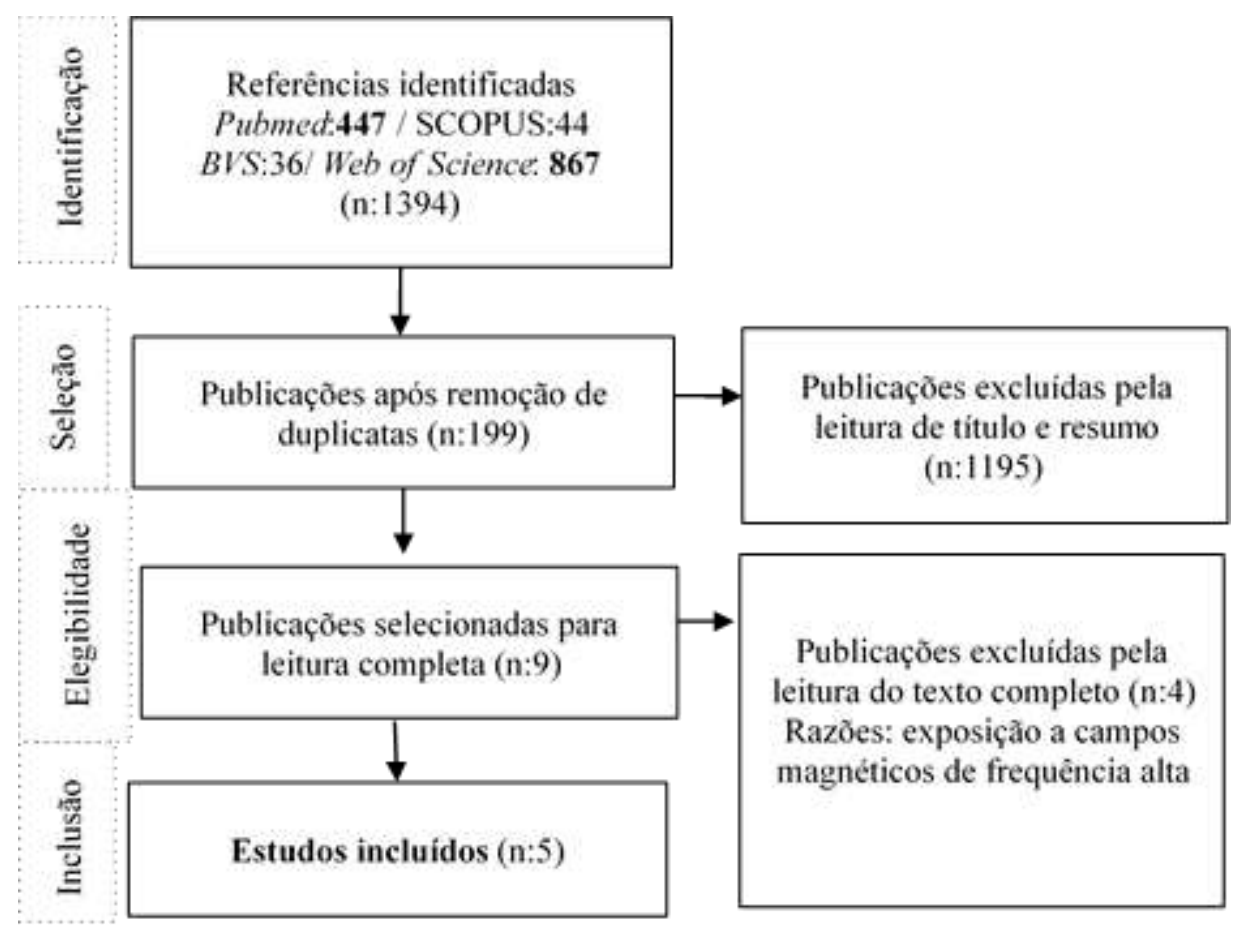

Fonte: Autores.

No Quadro 2 está apresentada a caracterização dos cinco estudos relacionados ao risco de leucemia e a exposição ELF-ML, segundo as variáveis primeiro autor/ano e revista da publicação, país e faixa etária dos participantes, grupos casos e controles, desfecho, conclusões e limitações dos estudos.

Quadro 2. Caracterização dos estudos relacionados ao risco de leucemia e a exposição ELF-ML (n=5).

\begin{tabular}{|c|c|c|c|}
\hline $\begin{array}{c}\text { Primeiro Autor/Ano } \\
\text { Periódico }\end{array}$ & $\begin{array}{c}\text { País e faixa etária dos } \\
\text { participantes } \\
\text { Tamanho da amostra }\end{array}$ & Desfecho & Conclusão e limitações \\
\hline $\begin{array}{l}\text { NUNEZ-ENRIQUEZ, } \\
2020 \\
\text { Bioelectromagnetics } \\
\text { Society }\end{array}$ & $\begin{array}{l}\text { - México } \\
\text { - } 0 \text { a } 16 \text { anos } \\
\text { Grupo Controle (n:407) } \\
\text { Grupo Caso (n:297) }\end{array}$ & $\begin{array}{l}\text { Investigar a associação } \\
\text { entre a exposição a } \\
\text { ELF-ML e o risco de } \\
\text { leucemia linfoblástica } \\
\text { aguda linhagem B }\end{array}$ & $\begin{array}{l}\text { Exposição a ELF-ML }(\geq 0,4 \mu \mathrm{T}) \text { pode estar } \\
\text { associada ao risco de desenvolvimento de } \\
\text { leucemia. Limitações: Não analisado a influência } \\
\text { de fontes externas. Tempo curto de avaliação ( } 24 \\
\text { horas). Não foi avaliado o papel das primeiras } \\
\text { infecções como fator de risco. É possível que } \\
\text { outros fatores relacionados a exposição de campos } \\
\text { magnéticos que não pode ser identificado, seja } \\
\text { mais relevante como para o desenvolvimento de } \\
\text { leucemia infantil. }\end{array}$ \\
\hline $\begin{array}{l}\text { KHEIFETS, } 2017 \\
\text { Cancer Causes \& } \\
\text { Control }\end{array}$ & $\begin{array}{l}\text { - Estados Unidos da } \\
\text { América } \\
\text { - Menos de } 16 \text { anos } \\
\text { Grupo Controle (n:5788) } \\
\text { Grupo Caso (n:5788) } \\
\end{array}$ & $\begin{array}{l}\text { Registro do risco de } \\
\text { leucemia e a exposição } \\
\text { a ELF-ML na } \\
\text { Califórnia }\end{array}$ & $\begin{array}{l}\text { Não fornece evidências claras sobre os riscos } \\
\text { associados a maiores exposições a ELF-ML. } \\
\text { Limitações: Falta de dados para outros potenciais } \\
\text { riscos de exposição. }\end{array}$ \\
\hline $\begin{array}{l}\text { PEDERSEN, } 2015 \\
\text { British Journal of Cancer }\end{array}$ & $\begin{array}{l}\text { - Dinamarca } \\
\text { - Menos de } 15 \text { anos } \\
\text { Grupo Controle (n:9129) } \\
\text { Grupo Caso (n:3277) }\end{array}$ & $\begin{array}{l}\text { Estender um estudo } \\
\text { realizado } \\
\text { anteriormente com } \\
\text { crianças } \\
\text { diagnosticadas com } \\
\text { leucemia, tumores de } \\
\text { SNC e linfoma } \\
\text { maligno entre } 1987 \text { e } \\
2003 \\
\end{array}$ & $\begin{array}{l}\text { Não encontraram evidências, referente a um risco } \\
\text { maior para leucemia, tumor do SNC e linfoma ao } \\
\text { incluir dados recente. } \\
\text { Limitações: Baixo número de casos expostos } \\
\text { devido à baixa prevalência da exposição no estudo } \\
\text { e a baixa incidência de câncer infantil. }\end{array}$ \\
\hline
\end{tabular}




\begin{tabular}{|c|c|c|c|}
\hline $\begin{array}{l}\text { SALVAN, } 2015 \\
\text { International Journal of } \\
\text { Environmental Research } \\
\text { and Public Health }\end{array}$ & $\begin{array}{l}\text { - Itália } \\
\text { - } 0 \text { a } 10 \text { anos } \\
\text { Grupo Controle (n:1380) } \\
\text { Grupo Caso (n:713) }\end{array}$ & $\begin{array}{l}\text { Exposição a ELF-ML } \\
\text { e associação a um } \\
\text { risco aumentado de } \\
\text { leucemia infantil }\end{array}$ & $\begin{array}{l}\text { Não foi evidenciada associação entre exposição- } \\
\text { doença. Limitações: dificuldade nas medições de } \\
\text { exposição estimada, viés de participação, erros de } \\
\text { avaliação de exposição. }\end{array}$ \\
\hline $\begin{array}{l}\text { JIRIK, } 2012 \\
\text { Biomedical and } \\
\text { Environmental Sciences }\end{array}$ & $\begin{array}{l}\text { - República Checa } \\
\text { - Menos de } 15 \text { anos } \\
\text { Grupo Controle (n:81) } \\
\text { Grupo Caso (n:82) }\end{array}$ & $\begin{array}{l}\text { Exposição a ELF-ML } \\
\text { e associação a um } \\
\text { risco aumentado de } \\
\text { leucemia infantil }\end{array}$ & $\begin{array}{l}\text { Não foi encontrada qualquer associação entre } \\
\text { níveis de exposição e leucemia infantil. } \\
\text { Limitações: Quantidade pequena de participantes. }\end{array}$ \\
\hline
\end{tabular}

Fonte: Autores.

A população amostral foi composta em quatro estudos por casos de leucemia infantil (Núnez-Enríquez et al., 2020; Kheifets et al., 2017; Salvan et al., 2015; Jirik et al., 2012) e apenas um estudo utilizou além do diagnóstico de leucemia infantil, casos com tumores de sistema nervoso central e linfoma (Pedersen et al., 2015).

Nos estudos, os controles elegíveis não possuíam diagnóstico de câncer. Dois estudos (Núnez-Enríquez et al., 2020; Jirik et al., 2012) selecionaram casos provenientes de hospitais para tratamento de doenças que não fosse leucemia infantil, e em outros três (Kheifets et al., 2017; Pedersen et al., 2015; Salvan et al., 2015) os casos foram da comunidade.

As variáveis gênero e idade foram usadas como critério de pareamento dos controles em quatro estudos (NúnezEnríquez et al., 2020; Kheifets et al., 2017; Pedersen et al., 2015; Salvan et al., 2015) e apenas um (Jirik et al., 2012) considerou a idade e a residência fixa para parear. Quanto à idade dos participantes, em dois estudos foi de menos de 16 anos (Núnez-Enríquez et al., 2020; Kheifets et al., 2017), outros dois (Pedersen et al., 2015; Jirik et al., 2012) menos de 15 anos e em um (Salvan et al., 2015) a idade variou de 0 a 10 anos.

Referente às informações para recrutar os casos e controles, três estudos (Kheifets et al., 2017; Pedersen et al., 2015; Salvan et al., 2015) utilizaram banco de dados populacionais e dois (Núnez-Enríquez et al., 2020; Salvan et al., 2015) usaram hospitais onde os participantes do estudo eram atendidos, sendo os casos pacientes em tratamento para leucemia e controles outros atendimentos não oncológicos.

Com relação à avaliação da exposição aos campos magnéticos, os estudos selecionados utilizaram diferentes abordagens, dois (Núnez-Enríquez et al., 2020; Salvan et al., 2015) realizaram as medições na residência dos participantes do estudo, no quarto na proximidades da cama, no período de 24 horas; dois (Kheifets et al., 2017; Pedersen et al., 2015) utilizaram a distância da linha de transmissão dos campos magnéticos até a residência, e apenas um (Jirik et al., 2012) utilizou a medição entre os campos magnéticos e a residência e desta até a escola dos participantes do estudo.

Todos os estudos avaliados encontraram limitações, tais como a não avaliação de outras fontes externas, como, por exemplo, a exposição a fios de alta tensão, poluição do ar, pesticidas (Núnez-Enríquez et al., 2020; Kheifets et al., 2017); baixo número de participantes (Pedersen et al., 2015; Jirik, et al., 2012) e dificuldade na medição (Salvan et al., 2015). Um estudo (Núnez-Enríquez et al., 2020) considerou que o tempo de exposição avaliado nas 24 horas foi curto e que a não avaliação das ocorrências das primeiras infecções é também um limitador.

Os métodos desenvolvidos nos estudos foram heterogêneos para a avaliação da exposição ao ELF-ML, ambientes analisados e os critérios utilizados para tal, o que não permite uma análise robusta em uma revisão.

Os estudos desta revisão demonstraram que embora os participantes do estudo estiveram expostos frequentemente a ELF-ML, não existem evidências robustas de que estas exposições sejam consideradas exclusivamente como fatores de risco para o desenvolvimento de leucemia infantil, pois não foi possível excluir outros fatores externos, os quais também podem estar associados ao desenvolvimento da doença.

Esta condição de limitação dos estudos foi descrita em uma revisão sistemática que analisou a exposição de radiação não ionizante e a relação com a leucemia infantil. Concluiu que todos os estudos analisados sofreram influência de variáveis 
externas, as quais interferiram nos resultados (Calvente et al., 2010). Este fato também foi evidenciado em estudos conduzidos em países de média e baixa renda (Baaken et al., 2020).

Embora venha ser relatado que a exposição residencial a ELF-ML pode levar ao desenvolvimento de leucemia infantil, a contribuição para tal é considerada pequena e atualmente caracterizada como incerta em decorrência dos estudos existentes (Grellier et al., 2014).

Desta forma, enquanto as variáveis de confusão não forem totalmente controladas, poderão ser apontadas como explicações alternativas encontradas nas associações ou não dos estudos de caso-controle que relacionam a exposição à radiação não ionizante ELF-ML e a leucemia infantil (Pelissari et al., 2009).

A correta quantificação da exposição e a ausência de um mecanismo que esclareça a interação entre os campos magnéticos e os reais efeitos na saúde integram as dificuldades para averiguar os riscos à saúde relacionados a campos eletromagnéticos (Marcilio et al., 2009).

Observa-se que em situações reais, as pessoas estão simultaneamente submetidas aos campos eletromagnéticos de fontes diferentes, e este efeito biológico de múltiplas fontes e múltiplas frequências ainda precisa ser explorado, visto que até agora não existem informações disponíveis (Saliev et al., 2018).

A realização de novas pesquisas sobre o tema é necessária, pois existe uma preocupação pública de que a exposição a campos eletromagnéticos de frequência extremamente baixa, a longo prazo, possa ser maléfica a saúde. Existem evidências sugestivas de exposição pré-natal e risco aumentado de leucemia e câncer cerebral na criança (Auger et al., 2019), além de riscos para os adultos no desenvolvimento de cânceres de cérebro e mama (Carpenter, 2019). Este tema necessita ser melhor elucidado, com subsídios para a tomada de decisões, os futuros estudos devem propor medições baseadas em protocolos predefinidos e relatórios padronizados de forma sistemática para gerar conclusões assertivas e minimizar incertezas.

Este estudo apresenta potencialidades com relação à busca detalhada e rigorosa dos artigos, assim como o desenvolvimento do método, mesmo assim as limitações estão relacionadas a ausência de uma análise estatística robusta em decorrência da variabilidade dos dados.

\section{Considerações Finais}

A partir da busca realizada, esta revisão integrativa foi desenvolvida de modo pontual com estudos de caso-controle. Ficou evidente que não foi possível evidenciar a existência de relação entre a presença ELF-MF e o desenvolvimento de leucemia infantil, em decorrência da heterogeneidade metodológico de mensuração, tempo de exposição e local de exposição o que indica fatores de confundimento. Assim, existe a necessidade de estudos com maior robustez para pautar conclusões e tomadas de decisão sobre os riscos ou não da exposição infantil ao ELF-MF.

\section{Agradecimentos}

Agradecemos ao programa de Pós-graduação em Saúde e Desenvolvimento na Região Centro-Oeste da Universidade Federal de Mato Grosso do Sul e o seu corpo docente pela contribuição e incentivo no desenvolvimento da pesquisa

\section{Referências}

Auger, N., Bilodeau-Bertrand, M., Marcoux, S., \& Kosatsky, T. (2019). Residential exposure to electromagnetic fields during pregnancy and risk of child cancer: A longitudinal cohort study. Environmental research, 176, 108524. https://doi.org/10.1016/j.envres.2019.108524

Belson, M., Kingsley, B., \& Holmes, A. (2007). Risk factors for acute leucemia in children: a review. Environmental Health Perspectives, 115(1), 138-145. https://doi.org/10.1289/ehp.9023 
Calvente, I., Fernandez, M. F., Villalba, J., Olea, N., \& Nuñez, M. I. (2010). Exposure to electromagnetic fields (non-ionizing radiation) and its relationship with childhood leukemia: A systematic review. Science of the Total Environment, 408(16), 3062-3069. https://doi.org/10.1016/j.scitotenv.2010.03.039

Carpenter, D. O. (2019). Extremely low frequency electromagnetic field cancer: How source of funding affects results. Environmental Reserch, 178, 108688. https://doi.org/10.1016/j.envres.2019.108688

Coebergh, J. W. W., Reedij, A. M. J., De Vries, E., Martos, C., Jakab, Z., Steliarova-Foucher, E., \& Kamp, W. A. (2006). Leukemia incidence and survival in children and adolescents in Europe during 1978-1997. Report from the automated childhood cancer information system project. European Journal of Cancer, 42, 2019-2036. https://doi.org/10.1016/j.ejca.2006.06.005

Curtin. K., Smith, K. R., Fraser, A., Pimentel, R., Kohlmann, W., \& Schiffman, J. D. (2013). Familial risk of childhood cancer and tumors in the Li-Fraumeni spectrum in the Utah Population Database: Implications for genetic evaluation in pediatric practice. The International Journal of Cancer, 133(10): 24442453. https://doi.org/10.1002/ijc.28266

Diab, K. A. (2020). The impact of the low frequency of the electromagnetic field on human. Advances in Experimental Medicine and Biology, 1237, 135-149. https://doi.org/10.1007/5584_2019_420

Donato, H., \& Donato, M. (2019). Etapas na condução de uma revisão sistemática. Acta Médica Portuguesa, 32(3), 227-235. https://doi.org/10.20344/amp.11923

Greenland, S., Sheppard, A. R., Kaune, W. T., Poole, C., \& Kelsh, M. A. (2000). A pooled analysis of magnetic fields, wire codes, and childhood leukemia. Epidemiology, 11(6), 624-634. https://doi.org/10.197/00001648-200011000-00003

Grellier, J., Ravazzani, P., \& Cardis, E. (2014) Potential health impacts of residential exposure to extremely low frequency magnetic fields in Europe. Environment International, 62, 55-63. https://doi.org/10.1016/j.envint.2013.09.017

Instituto Nacional de Câncer José Alencar Gomes da Silva. Tipos de câncer (21019). Rio de Janeiro: INCA. https://www.inca.gov.br/tipos-de-cancer.

International Agency for Research on Cancer (2002). Non-Ionizing radiation, Part 1: Static and extremely low-frequency (ELF) electric and magnetic. IARC Monographs Evalution Carcinogenic Risk to Human, 80.

Jin, M. W., Xu, S. M., An, Q., \& Wang, P. (2016). A review of risk factors for childhood leucemia. European Review for Medical and Pharmacological Sciences, 20(18), 3760-3764. https://www.europeanreview.org/article/11444

Jirik, V., Pekarek, L., Janout, V., \& Tomaskova, H. (2012). Association between childhood leukaemia and exposure to power-frequency magnetic Fields in Middle Europe. Biomedical and Environmental Sciences, 25(5), 597-601. https://doi: 10.3967/0895-3988.2012.05.015

Karimi, A., Moghaddam, F. G., \& Valipour, M. (2020). Insights in the biology of extremely low-frequency magnetic Fields exposure on human health. Molecular Biology Reports, 47(7), 5621-5633. https://doi.org/10.1007/s11033-020-05563-8

Kaszuba-Zwoinska, J., Gremba, J., Galdzinska-Calik, B., Wójcik-Piotrowicz, K., \& Thor, J. P. (2015). Electromagnetic field induced biological effects in humans. Przegl Lek, 72(11), 636-641. https://pubmed.ncbi.nlm.nih.gov/27012122/

Kheifets, L., Crespi, C. M., Hooper, C., Cockburn, M., Amonn, A. T., \& Vergara, X. P. (2017). Residential magnetic fields exposure and childhood leukemia: a population-based case-control study in California. Cancer Causes \& Control, 28(10), 1117-1123. https://doi.org/10.1007/s10552-017-0951-6

Maia, R. R. P. \& Filho, V. W. (2013). Infection and childhood leukemia: review of evidence. Revista de Saúde Pública, 47(6), 1172-1185. https://doi.org/10.1590/S0034-8910.2013047004753

Marcilio, I., Habermann, M., \& Gouveia, N. (2009). Campos magnéticos de frequência extremamente baixa e efeitos na saúde: revisão de literatura. Revista Brasileira de Epidemiologia, 12(2), 105-123. https://doi.org/10.1590/s1415-790x2009000200002

Moher, D., Liberati, A., Tetzlaff, J., Altman, D. G., \& The Prisma Group. (2009). Preferred reporting items for systematic reviews and meta-analyses: the PRISMA statement. PLoS Medicine, 6(7), 1-6. https://doi.org/10.1371/journal.pmed.1000097

Núñez-Enríquez, J. C., Correa-Correa, V., Flores-Lujano, J., Pérez-Saldivar, M. L., Jiménez-Hernández, E., Martín-Trejo, J. A., Espinoza-Hernández, L. E., Medina-Sanson, A., Cárdenas-Cardos, R., Flores-Villegas, L. V., Peñaloza-González. J. G., Torres-Nava, J. R., Espinosa-Elizondo, R. M., Amador-Sánchez, R., Rivera-Luna, R., Dosta-Herrera, J. J., Mondragón-Garcia, J. A., González-Ulibarri, J. E., Martínez-Silva, S. I., Espinoza-Anrubio, G., Duarte-Rodríguez, D. A., García-Cortés, L. R., Gil-Hernández, A. E., \& Mejía-Aranguré, J. M. (2020). Extremely low-frequency magnetic fields and the risk of childhood B-lineage acute lymphoblastic leukemia in a city with high incidence of leukemia and elevated exposure to ELF Magnetic Fields. Bioelectromagnetics, 41(8), 581-597. http://doi.org/10.1002/bem.22295

Ouzzani, M., Hammady, H., Fedorowicz, Z., \& Elmagarmid, A. (2016). Rayyan—a web and mobile app for systematic reviews. Systematic Reviews, 5(1). https://doi.org/10.1186/s13643-016-0384-4

Pedersen, C., Raaschou-Nielsen, O., Rod, N. H., Frei, P., Poulsen, A. H., Johansen, C., \& Schu, J. (2014). Distance from residence to power line and risk of childhood leukemia: a populationbased case-control study in Denmark. Cancer Causes Control, 25(2), 171-177. https://doi.org/10.1007/s10552-013-0319-5

Pedersen, C., Johansen, C., Schuz, J., Olsen, J. H., \& Raaschou-Nielsen, O. (2015). Residential exposure to extremely low-frequency magnetic fields and risk of childhood leukaemia, CNS tumour and lymphoma in Denmark. British Journal of Cancer, 13, 1370-1374. http://doi.org/10.1038/bjc.2015.365

Pelissari, D. M., Barbieri, F. E., \& Wünsch Filho, V. (2009). Magnetic Fields and acute lymphoblastic leucemia in children: a systematic review of casecontrol studies. Caderno de Saúde Pública, 25(3), S441-S452. https://doi.org/10.1590/S0102-311X2009001500009

Salvan, A., Ranucci, A., Lagorio, S., Magnani, C., \% Em nome do SETIL Research Group. (2015). Childhood leucemia and 50 Hz magnetic Fields: findings from the Italian SETIL case-control study. International Journal of Environmental Research and Public Health, 12(2), 2184-2204. https://doi.org/10.3390/ijerph120202184 
Research, Society and Development, v. 10, n. 12, e19101320745, 2021

(CC BY 4.0) | ISSN 2525-3409 | DOI: http://dx.doi.org/10.33448/rsd-v10i13.20745

Saliev, T., Begimbetova, D., Masoud, A., \& Matkarimov, B. (2018). Biological effects of non-ionizing electromagnetic field: Two sides of a coin. Progress in Biophysics and Molecular Biology, 141, 25-36. https://doi.org/10.1016/j.pbiomolbio.2018.07.009

Scarfi, M. R., Mattsson, M., Simkó, M., \& Zeni, O. (2019). Special Issue: "Electric, Magnetic, and Electromagnetic Fields in Biology and Medicine: From Mechanisms to Biomedical Applications". International Journal of Environmental Research and Public Health, 16(22), 4548. https://doi.org/10.3390/ijerph16224548

Scientific Committee on Emerging Newly Identified Health Risks (2015). Opinion on potential Health Effects of Exposureto Electromagnetic Fields. Bioelectromagnetics, 36(6), 480-484. https://doi.org/10.1002/bem.21930

Stillwell, S., Fineoutr-Overholt, E., Melnyk, B., \& Wiliiamson, K. (2010). Evidence-based practice, step by step: searching for the evidence. Journal of Advanced Nursing, 110(5), 41-47. https://doi.org/10.1097 / 01.NAJ.0000372071.24134.7e

Wertheimer, N., \& Leeper, E. (1979). Electrical wiring configurations and childhood cancer. American Journal of Epidemiology, 109(3), 273-284. https://doi.org/10.1093/oxfordjournals.aje.a112681

Whitehead, P., Metayer, C., Wiemels, J. L., Singer, A, W., \& Miller, M. D. (2016). Childhood Leukemia and Primary Prevention. Current Problems Pediatric Adolescent Health Care, 46(10), 317-352. https://doi.org/10.1016/j.cppeds.2016.08.004

Whittemore, R., \& Knafl, K. (2005). The integrative review: updated methodology. Journal of Advanced Nursing, 52(5), 546-553. https://doi.org/10.1111/j.1365-2648.2005.03621.x 